\title{
A Lagrangian for von Karman equations of large deflection problem of thin circular plate
}

\author{
Ji-Huan $\mathrm{He}^{*}$ \\ LNM, Institute of Mechanics, Chinese Academy of Sciences, Beijing 100080, China \\ Shanghai Institute of Applied Mathematics and Mechanics, 149 Yanchang Road, \\ Shanghai 200072, China
}

\begin{abstract}
By the semi-inverse method proposed by He, a Lagrangian is established for the large deflection problem of thin circular plate. Ritz method is used to obtain an approximate analytical solution of the problem. First order approximate solution is obtained, which is similar to those in open literature. By Mathematica a more accurate solution can be deduced.

(C) 2002 Elsevier Science Inc. All rights reserved.

Keywords: von Karman equations; Plate; Variational principle; Semi-inverse method; Ritz method
\end{abstract}

\section{Introduction}

Though the fast development of various numerical technologies by means of high-speed computer, the analytical approaches to a physical problem, however, continue to keep their own primary importance and will become more and more important. The numerical solutions can reveal the numerical feature of the problem, but they can not reveal the important physical features involved in the equations. For example, numerical methods can solve a nonlinear

\footnotetext{
*Address: College of Science, Shanghai Donghua University, P.O. Box 471, 1882 Yan'an Xilu Road, 200051 Shanghai, China.

E-mail addresses: jhhe@dhu.edu.cn, ijnsns@yahoo.com.cn (J.-H. He).
} 
oscillation equation without any difficulties, but the solution can not reveal the inherent characteristics of dependence of the nonlinear angular frequency upon initial conditions. The analytical approach, in the other hand, provides physical insight into the nature of the solution of the problem.

Recently various analytical methods are proposed to solve nonlinear equations, for example, variational iteration method [1], homotopy perturbation method [2], a new perturbation technique depending upon an artificial parameter [3], an iteration perturbation [4], where the numerical technique is coupled with the classic perturbation method, resulting in a powerful tool, and so on. A review on these methods can be found in details in Ref. [5]. In this paper, we will use the Ritz method to solve von Karman equations for large deflection problem of thin circular plate, to this end, we will apply the semiinverse method proposed by $\mathrm{He}[6,7]$ to search for the required variational functional.

\section{The von Karman equations}

The large deflection problem of thin circular plate under uniformly loaded pressure can be treated by means of von Karman equations, which can be written in dimensionless form as [8-12]

$$
\begin{aligned}
& S_{\mathrm{t}}=S_{\mathrm{r}}+2 x \frac{\mathrm{d} S_{\mathrm{r}}}{\mathrm{d} x}, \\
& \frac{\mathrm{d}^{2}}{\mathrm{~d} x^{2}}\left(x S_{\mathrm{r}}\right)+\frac{1}{2}\left(\frac{\mathrm{d} w}{\mathrm{~d} x}\right)^{2}=0, \\
& \frac{3}{16} P+\frac{3}{4}\left(1-v^{2}\right) S_{\mathrm{r}} \frac{\mathrm{d} w}{\mathrm{~d} x}-\frac{1}{4} \frac{\mathrm{d}^{2}}{\mathrm{~d} x^{2}}\left(x \frac{\mathrm{d} w}{\mathrm{~d} x}\right)=0 .
\end{aligned}
$$

The dimensionless quantities $W(x), P, S_{\mathrm{r}}(x)$ and $S_{\mathrm{t}}(x)$ are defined, respectively, as

$$
W(x)=\frac{w}{h}, \quad P=\frac{a^{4} q}{h^{4} E}\left(1-v^{2}\right), \quad S_{\mathrm{r}}(x)=\frac{a^{2}}{E h^{3}} N_{\mathrm{r}}, \quad S_{\mathrm{t}}(x)=\frac{a^{2}}{E h^{3}} N_{\mathrm{t}},
$$

and the dimensionless coordinate $x$ is defined as $x=r^{2} / a^{2}, 0 \leqslant x \leqslant 1$.

Here $h, a$ are the plate thickness and the plate radius, respectively, $q, E$ are the uniform load density and Young's modulus of the plate material, respectively, $v$ is Poisson's ratio, $r$ is the radial coordinate, $N_{\mathrm{r}}, N_{\mathrm{t}}$ are the radial and tangential membrane force, respectively, $w$ is the normal displacement of the plate at distance $r$ from the center of the plate.

Many researchers had done this problem by various different methods. A tedious numerical computation was proposed by Way [8] by means of power 
series, and Levy [9] applied the trigonometric series to solve a rectangular plate. Both the above methods involve the numerical determination of an infinite number of coefficients, and hence the number of equations are excessive, thus rendering these methods prohibitively expensive, if not altogether impractical, for routine application purposes. To alleviate the above drawback, Chien $[10,11]$ proposed a perturbation technique, where he used the ratio of the center deflection to the thickness as a small parameter, the method is now called the composite expansion, and was generalized in Ref. [12].

\section{Variational principle}

In this paper we will use direct variational methods to search for an analytical solution for the problem. The direct variational methods have at least the following two advantages [13]: (1) they provide physical insight into the nature of the solution of the problem; (2) they generally have a higher rate of asymptotic convergence and result in a much smaller system of equations. To this end, we will apply the semi-inverse method $[6,7]$ to the establishment of a variational principle for the problem.

The essence of the semi-inverse method is to construct a trial functional with an unknown function $F$. For the present problem we can construct the following functional with two fields $S_{\mathrm{r}}$ and $W$ :

$$
J\left(S_{\mathrm{r}}, W\right)=\int_{0}^{1} L\left(S_{\mathrm{r}}, W\right) \mathrm{d} x,
$$

where $L\left(S_{\mathrm{r}}, W\right)$ is a Lagrangian.

Supposing the stationary conditions of the above functional satisfy the field Eqs. (2) and (3). In order to search for such a functional, we begin with the following Lagrangian

$$
L\left(S_{\mathrm{r}}, W\right)=-\frac{1}{2}\left[\frac{\mathrm{d}}{\mathrm{d} x}\left(x S_{\mathrm{r}}\right)\right]^{2}+\frac{1}{2} x S_{\mathrm{r}}\left(\frac{\mathrm{d} W}{\mathrm{~d} x}\right)^{2}+F(W),
$$

where $F(W)$ is an unknown function of $W$ and its derivatives.

There exist various approaches to the establishment of trial functionals for a physical problem, illustrative examples can be found in Refs. [14-19].

The above advantage of the trial functional lies on the fact that the stationary condition with respect to $S_{\mathrm{r}}$ is

$$
\frac{\partial L}{\partial S_{\mathrm{r}}}-\frac{\partial}{\partial x} \frac{\partial L}{\partial\left(\partial S_{\mathrm{r}} / \partial x\right)}=0
$$


i.e.

$$
x \frac{\mathrm{d}^{2}}{\mathrm{~d} x^{2}}\left(x S_{\mathrm{r}}\right)+\frac{1}{2} x\left(\frac{\mathrm{d} W}{\mathrm{~d} x}\right)^{2}=0 .
$$

In case $x \neq 0$, the above equation is equivalent to the field Eq. (2). The stationary condition with respect to $W$ reads

$$
-\frac{\mathrm{d} W}{\mathrm{~d} x}\left(x S_{\mathrm{r}} \frac{\mathrm{d} W}{\mathrm{~d} x}\right)+\frac{\delta F}{\delta W}=0,
$$

where $\delta F / \delta W$ is called variational derivative defined as

$$
\frac{\delta F}{\delta W}=\frac{\mathrm{d} L}{\mathrm{~d} W}-\frac{\mathrm{d}}{\mathrm{d} x} \frac{\partial L}{\partial(\mathrm{d} W / \mathrm{d} x)}+\frac{\mathrm{d}^{2}}{\mathrm{~d} x^{2}} \frac{\partial L}{\partial\left(\mathrm{d}^{2} W / \mathrm{d} x^{2}\right)}-\cdots
$$

We search for such an $F$, so that Eq. (8) turns out to be Eq. (3). Compared with Eq. (8), the field Eq. (3) should be made such a modification so that the unknown $F$ in (8) can be easily identified.

Multiplying Eq. (3) by $4 x /\left(3\left(1-v^{2}\right)\right)$, then differentiating the obtained equation with respect to $x$, we have the following equation

$$
\frac{1}{4\left(1-v^{2}\right)} P+\frac{\mathrm{d}}{\mathrm{d} x}\left(x S_{\mathrm{r}} \frac{\mathrm{d} W}{\mathrm{~d} x}\right)-\frac{1}{3\left(1-v^{2}\right)} \frac{\mathrm{d}}{\mathrm{d} x}\left[x \frac{\mathrm{d}^{2}}{\mathrm{~d} x^{2}}\left(x \frac{\mathrm{d} W}{\mathrm{~d} x}\right)\right]=0 .
$$

We set

$$
\begin{aligned}
\frac{\delta F}{\delta W} & =\frac{\mathrm{d} W}{\mathrm{~d} x}\left(x S_{\mathrm{r}} \frac{\mathrm{d} W}{\mathrm{~d} x}\right) \\
& =-\frac{1}{4\left(1-v^{2}\right)} P+\frac{1}{3\left(1-v^{2}\right)} \frac{\mathrm{d}}{\mathrm{d} x}\left[x \frac{\mathrm{d}^{2}}{\mathrm{~d} x^{2}}\left(x \frac{\mathrm{d} W}{\mathrm{~d} x}\right)\right]
\end{aligned}
$$

in Eq. (8), so that Eq. (8) satisfies (9). From (10), the unknown $F$ can be readily identified as

$$
F=-\frac{1}{4\left(1-v^{2}\right)} P W+\frac{1}{6\left(1-v^{2}\right)}\left[\frac{\mathrm{d}}{\mathrm{d} x} \times \frac{\mathrm{d}}{\mathrm{d} x} W\right]^{2} .
$$

Substituting (11) into (5), we obtain the required Lagrangian, which reads

$$
\begin{aligned}
L\left(S_{\mathrm{r}}, W\right)= & -\frac{1}{2}\left[\frac{\mathrm{d}}{\mathrm{d} x}\left(x S_{\mathrm{r}}\right)\right]^{2}+\frac{1}{2} x S_{\mathrm{r}}\left(\frac{\mathrm{d} W}{\mathrm{~d} x}\right)^{2} \\
& +\frac{1}{6\left(1-v^{2}\right)}\left[\frac{\mathrm{d}}{\mathrm{d} x} x \frac{\mathrm{d}}{\mathrm{d} x} W\right]^{2}-\frac{1}{4\left(1-v^{2}\right)} P W .
\end{aligned}
$$




\section{Ritz method}

In the above section we success in obtaining a necessary variational principle for the discussed problem, in this section we will apply direct variational methods to the problem. The direct variational methods such as Ritz and Kantorovitch techniques have been, and continue to be, popular tools for nonlinear analysis.

Consider the case of fixed boundary, the boundary conditions can be written as

$$
\begin{aligned}
& W=\frac{\mathrm{d} W}{\mathrm{~d} x}=0 \quad \text { at } x=1, \\
& 2 \frac{\mathrm{d} S_{\mathrm{r}}}{\mathrm{d} x}+(1-v) S_{\mathrm{r}}=0 \quad \text { at } x=1, \\
& S_{\mathrm{r}} \quad \text { and } \frac{\mathrm{d} W}{\mathrm{~d} x} \quad \text { remain finite at } x=0 .
\end{aligned}
$$

We choose the following trial functions which satisfy the boundary conditions:

$$
\begin{aligned}
& W=A(1-x)^{2}, \\
& S_{\mathrm{r}}=B(C+x),
\end{aligned}
$$

where $A, B$ are unknown constants, and $C=-(3-v) /(1-v)$.

Substituting (16) and (17) into (12), we have

$$
\begin{aligned}
L(A, B)= & -\frac{1}{2} B^{2}(C+2 x)^{2}+2 B A^{2} x(C+x)(1-x)^{2}+\frac{4 A^{2}}{6\left(1-v^{2}\right)}(1-2 x)^{2} \\
& -\frac{1}{4\left(1-v^{2}\right)} P A(1-x)^{2} .
\end{aligned}
$$

So the functional (4) now becomes

$$
\begin{aligned}
J(A, B)= & \int_{0}^{1} L(A, B) \mathrm{d} x=-\frac{1}{12} B^{2}\left[(C+2)^{3}-C^{3}\right]+2 B A^{2} \\
& \times\left(\frac{C}{2}+\frac{1-2 C}{3}+\frac{C-2}{4}+\frac{1}{5}\right)+\frac{2 A^{2}}{9\left(1-v^{2}\right)}-\frac{1}{12\left(1-v^{2}\right)} P A .
\end{aligned}
$$

The stationary conditions require that

$$
\begin{aligned}
& \frac{\partial J}{\partial A}=4 B A\left(\frac{5 C-2}{60}\right)+\frac{4 A}{9\left(1-v^{2}\right)}-\frac{1}{12\left(1-v^{2}\right)} P=0, \\
& \frac{\partial J}{\partial B}=-\frac{1}{6} B\left[(C+2)^{3}-C^{3}\right]+2 A^{2}\left(\frac{5 C-2}{60}\right)=0 .
\end{aligned}
$$


From (20), we obtain

$$
B=\frac{5 C-2}{5\left[(C+2)^{3}-C^{3}\right]} A^{2}=\frac{5 C-2}{10\left(3 C^{2}+4 C+4\right)} A^{2} .
$$

In view of (21), Eq. (19) can be rewritten as

$$
\frac{(5 C-2)^{2}}{150\left(3 C^{2}+4 C+4\right)} A^{3}+\frac{4}{9\left(1-v^{2}\right)} A-\frac{1}{12\left(1-v^{2}\right)} P=0 .
$$

Define $W_{\mathrm{m}}$ the maximal deflection at center, i.e. $W_{\mathrm{m}}=W(0)$. From (16) we know $A=W_{\mathrm{m}}$, so Eq. (22) can be written in the form

$$
\frac{3}{16} P=W_{\mathrm{m}}+\beta W_{\mathrm{m}}^{3}
$$

where

$$
\beta=\frac{3\left(1-v^{2}\right)(5 C-2)^{2}}{200\left(3 C^{2}+4 C+4\right)}, \quad C=-\frac{3-v}{1-v} .
$$

The relation (23) has been investigated approximated by a number of authors $[10,20,21]$. They results differ each in the values of $\beta$.

To improve the accuracy of the approximate solution, we can use the following trial functions

$$
\begin{aligned}
& W=A_{0}(1-x)^{2}\left(1+A_{1} x+A_{2} x^{2}+\cdots\right), \\
& S_{\mathrm{r}}=B_{0}(C+x)+(1-x)^{2}\left(B_{1}+B_{2} x+B_{3} x^{2}+\cdots\right),
\end{aligned}
$$

where $C=-(3-v) /(1-v)$, and $A_{i}$ and $B_{i}$ unknown constants, which can be determined from the following relations

$$
\begin{aligned}
& \frac{\partial J}{\partial A_{i}}=0 \quad(i=0,1,2, \ldots), \\
& \frac{\partial J}{\partial B_{i}}=0 \quad(i=0,1,2, \ldots) .
\end{aligned}
$$

The constants $A_{i}$ and $B_{i}$ can be easily determined by Mathematica.

\section{Conclusion}

In this paper we suggest another approach to the solution of the large deflection problem of thin circular plate by direct variational method. The variational principle is deduced by the semi-inverse method. The present paper aims at providing a more complete theoretical basis for the finite element applications, direct variational methods such as Ritz's, Trefftz's and Kantorovitch's methods. 


\section{References}

[1] J.H. He, Variational iteration method: a kind of nonlinear analytical technique: some examples, Int. J. Nonlinear Mech. 34 (4) (1999) 699-708.

[2] J.H. He, Homotopy perturbation technique, Comput. Meth. Appl. Mech. Eng. 178 (1999) $257-262$.

[3] J.H. He, A modified perturbation technique depending upon an artificial parameter, Meccanica 35 (2000) 299-311.

[4] J.H. He, Iteration perturbation method for strongly nonlinear oscillations, J. Vib. Control 7 (5) (2001) 631-642.

[5] J.H. He, A review on some new recently developed nonlinear analytical techniques, Int. J. Nonlinear Sci. Numer. Simul. 1 (1) (2000) 51-70.

[6] J.H. He, Semi-inverse method of establishing generalized variational principles for fluid mechanics with emphasis on turbomachinery aerodynamics, Int. J. Turbo Jet-Engines 14 (1) (1997) 23-28.

[7] J.H. He, Semi-inverse method and generalized variational principles with multi-variables in elasticity, Appl. Math. Mech. 21 (7) (2000) 797-808 (English Ed.).

[8] S. Way, Bending of circular plates with large deflection, ASME J. Appl. Mech. 56 (1934) 627636.

[9] S. Levy, Bending of rectangular plate with large deflections, NACA Report, No. 737, 1942.

[10] W.Z. Chien, Large deflection of a circular clamped plate under uniform pressure, Chin. J. Phys. 7 (2) (1947) 102-113.

[11] W.Z. Chien, Asymptotic behavior of a thin clamped circular plate under uniform normal pressure at very large deflection, The Science Reports of National Tsinghua University 5 (1) (1948) 71-94.

[12] W.Z. Chien, S.L. Chen, The solution of large deflection problem of thin circular plate by the method of composite expansion, Appl. Math. Mech. 6 (1) (1985) 25-49 (Chinese Ed.).

[13] A.K. Noor, Recent advances in the applications of variational methods to nonlinear problems, in: H. Kardestuncer (Ed.), Unification of Finite Element Methods, Elsevier Science Publisher B.V, North-Holland, 1984, pp. 275-302.

[14] J.H. He, Generalized Hellinger-Reissner principle, ASME J. Appl. Mech. 67 (2) (2000) 326331.

[15] J.H. He, A classical variational model for micropolar elastodynamics, Int. J. Nonlinear Sci. Numer. Simul. 1 (2) (2000) 133-138.

[16] J.H. He, Coupled variational principles of piezoelectricity, Int. J. Eng. Sci. 39 (3) (2000) $323-$ 341.

[17] J.H. He, Hamilton principle and generalized variational principles of linear thermopiezoelectricity, ASME J. Appl. Mech. 68 (4) (2001) 666-667.

[18] J.H. He, Comments on derivation and transformation of variational principles with emphasis on inverse and hybrid problems in fluid mechanics: a systematic approach, Acta Mech. 149 (14) (2001) 247-249.

[19] J.H. He, Inverse problems of determining the unknown shape of oscillating airfoils in compressible 2D unsteady flow via variational technique, Aircraft Eng. Aerospace Technol. 72 (1) (2000) 18-24.

[20] S. Timoshenko, in: Theory of Plates and Shells, McGraw Hill, 1940, pp. 333-337.

[21] A. McPherson, W. Ramberg, S. Levy, Normal pressure tests of circular plate with clamped edges, NACA Report, No. 744, 1942. 